\title{
LXIII. On the constitution of natural radiation
}

\section{J. Larmor F.R.S.}

To cite this article: J. Larmor F.R.S. (1905) LXIII. On the constitution of natural radiation, Philosophical Magazine Series 6, 10:59, 574-584, DOI: 10.1080/14786440509463404

To link to this article: http://dx.doi.org/10.1080/14786440509463404

$$
\text { 册 Published online: } 16 \text { Apr } 2009 .
$$

Submit your article to this journal

Џll Article views: 7

Q View related articles $\sqsubset$ 
Nickel-Steel 24.04 per cent.

\begin{tabular}{|c|c|c|c|c|c|}
\hline \multicolumn{2}{|c|}{$t=-2^{\circ} \cdot 0 \mathrm{C}$} & \multicolumn{2}{|c|}{$t=-64^{\circ} \cdot 0 \mathrm{C}}$. & \multicolumn{2}{|c|}{$t=-96^{\circ} \cdot 0 \mathrm{O}$} \\
\hline H. & $\frac{\delta l}{l} \times 10^{6}$ & H. & $\frac{\delta l}{l} \times 10^{6}$ & H. & $\frac{\delta l}{l} \times 10^{6}$ \\
\hline $\begin{array}{r}409 \\
124 \cdot 4 \\
266 \\
463\end{array}$ & $\begin{array}{l}0 \cdot 1 \\
0 \cdot 2 \\
0 \cdot 6 \\
1 \cdot 1\end{array}$ & $\begin{array}{r}43 \cdot 5 \\
114 \cdot 0 \\
217 \cdot 3 \\
402\end{array}$ & $\begin{array}{l}0.5 \\
20 \\
37 \\
66\end{array}$ & $\begin{array}{r}20.5 \\
44 \cdot 2 \\
125.4 \\
216.8 \\
318 \\
412\end{array}$ & $\begin{array}{l}0 \cdot 1 \\
1 \cdot 0 \\
3 \cdot 4 \\
5 \cdot 6 \\
7 \cdot 7 \\
9 \cdot 3\end{array}$ \\
\hline
\end{tabular}

From these numbers, we find a parallelism between the change of magnetization and that of the length-change. In weak fields, the change of length gradually increases as the temperature falls till it reaches a maximum, and then decreases. As the field becomes stronger the maximum elongation is displaced in lower temperatures, and at last vanishes. These changes are common to nickel-steels of percentages higher than 28.32 per cent.; for percentages lower than 26.64 per cent. the elongation for a consti:nt field at first increases gradually and then rapidly, soon approaching an asymptotic value as the temperature falls.

[To be continued.]

LXIII. On the Constitution of Natural Radiation. By J. LaRmor, F.R.S. *

THEE recent paper by Lord Rayleigh on "The Origin of 1 the Prismatic Colours" + recurs to fundamental and delicate points in the philosophy of Optics, first effectively expounded by himself in 1881 and the following years $\ddagger$, on which I desire to offer some observations; especially as the mode of exposition of the dispersive action of a prism which was adopted by me several years ago $\$$ has been the subject of criticism in the papers by Schuster and Ames, to which Lord Rayleigh refers at the beginning of his paper.

The first part of the following remarks would doubtless bear condensation, as the full force of Lord Rayleigh's comparison of the dispersion problem with that of a travelling maintained source had not been grasped when they were written ; but in a subject which so largely turns on the mode of expression, condensation might involve obscurity. There

* Communicated by the Author.

+ Phil. Mag. Oct. 1905, p. 401.

\pm Cf. especially 'Wave Theory' \$7, Ency. Brit. 1888; Scientific Papers, vol. iii.

\$ Fiher and Matter,' 1900, chap. xv. 
does not, however, seem to be in what follows anything that stands in definite contradiction with Lord Rayleigh's published views.

That there is room for still further precision of terminology in this subject is indeed suggested by the beginning of Lord Rayleigh's paper. It would seem that a definite choice can be made between the two modes of exposition, both of which he considered to be allowable. According to the first, "The assertion that Newton's experiments prove the colours to be already existent in white light is usually made in too unqualified a form." On a first impression this remark might be imagined to strike at the roots of all the various instrumental methods that have been elaborated for analysing complex radiations; for if the analysis brings out teatures that are not already existent in the radiation, two different methods of analysis (e. g., by a grating and by a prism) can hardly be expected to give concordant results.

The alternative mode of exposition is to say that each complex type of radiation is constituted definitely of those colours (simple trains of various wave lengths), into which the Fourier mathematical analysis wonld divide its vibrationcurve : and that various analysing instruments (gratings, prisms, \&c.) are capable of revealing this constitution with different amounts of precision, the outstanding differences between these analyses being treated as due to imperfections of the instruments as regards the purpose in question. This (the usual) point of view is claimed as a valid alternative in Lord Rayleigh's third paragraph : to hold, as is done infra, that in some cases the resulting analysis is so imperfect as to be valueless, need not disturb the general validity of this point of view.

So far, the matter is one as to the most suitable mode of theoretical description or formulation. But we presently reach questions on which opinions may perhaps differ as to physical fact. In the spectral analysis of ordinary coninuous radiation, the prism and the grating give consistent results, when well understood corrections and adaptations are applied before making the comparison. If these instruments are applied to radiation consisting of a system of sharp, entirely uncoordinated, discrete pulses, such as the Röntgen rays are supposed to be, will this general agreement continue? It is clear that the grating (if of ideal perfectly reflecting quality) will draw out each pulse into a spectrum, and thus will analyse the radiation. It seems open to question whether a prism will not merely gradually dissipate it by scattering, however wide the pulses may be, even if they are of breadth comparable with the wave-lengths of visible light. If this 
be so, the prism is very badly suited for the analysis of this type of radiation, and no amount of adaptation of the result will bring the prism into conformity with the ideal grating.

The Fourier mathematical process*, as also the ideal grating which reflects back the disturbance in échelon so to say, operates by simply selecting and piecing together elements existing in the original radiant disturbance, so as to isolate periodic wave-trains that on superposition would reproduce the form of the original vibration-curve.

On the other hand, what the prism may do to a given isolated pulse would seem to depend on its own constitution. The customary mode of investigation would be to replace the pulse by the equivalent infinite system of component Fourier wave-trains, to find the effect produced on each of them by substituting its expression in the differential dynamical equations of the dispersive medium, and to add the results thus found. Though the original Fourier expansion of the pulse is always analytically legitimate and definite, it is not always allowable, without scrutiny as to convergency, thus to operate on its separate terms and add. Indeed, the particular component waves whose period is a free period of the medium would increase infinitely in importance in the result : thus it must be ascertained whether this infinity of intensity is more than compensated by infinite smallness of the element of period over which it ranges, before the procedure which includes it can he accepted as mathematically legitimate. The insertion of a very small frictional term in the dynamical equation will, however, secure that the component vibration remains finite though great at this critical period, and the analysis then becomes entirely valid. But the problem is only shifted; it has now to be ascertained whether the limit which this solution approaches as the friction is reduced indefinitely is the same as the solution previously arrived at in the absolute absence of friction,-whether in fact there exists a definite limit. That there is in many cases no definite limit is merely another way of expressing the theory of anomalous or selective dispersion, in which the final steady result depends essentially on the magnitude of the small viscous term that must be introduced in order to evade infinities, while the mode of the gradual establishment of that result is likewise undetermined. The question thus arises, whether the proportion of the energy of an incident isolated pulse that goes into this selective vibration is capable of determination by operating analytically upon its Fourier analysis in this way, - whether, in fact, a different line of

* $C f$. ' Ether and Matter,' $\$ 162$. 
attack would not be required in order to determine it. At any rafe, such questions of mathematical validity can arise only in regard to the presence of selective or anomalous dispersion*.

The argument of Prof. Schuster (Phil. Mag. Jan. 1904, p. $6+$ ) arrives at the conclusion that a single pulse is split up regularly into a spectrum by a prism. It appears to start from an implied hypothesis that even an abrupt pulse travels unchanged across the dispersive medium, with the velocity appropriate to a group of waves. If for a pulse is substituted a train of waves with wave-lengths variable within the narrow limits $\lambda$ and $\lambda+\delta \lambda$, so that the train is very nearly simple harmonic, this statement will be sensibly exact except near the beginning and end of the train : and Prof. Schuster's representation of the emergent radiation, as consisting of groups of waves, most concentrated in the neighbourhood of surfaces which are oblique to the wave-fronts, then affords an instructive view of the process of dispersion, whether prismatic or diffractive. But if this argument is to be pressed so as to include a single sharp pulse, what value of $\lambda$ are we to take as applicable to it? The theorem of definite groupvelocity is demonstrated only for the compound disturbance arising from the superposition of simpler trains of some common type but with slightly differing parameters, - the trains being unlimited and of simple harmonic type in the usual Stokes-Rayleigh theory. A single pulse will thus not have any definite group-velocity : with which it can travel; or, what comes to the same, the different parts of it will travel in the dispersive medium with widely different velocities, so that it will spread ont and be dissipated. An argument which assigns a definite velocity to a complex disturbance can thus be applicable only to very special types of disturbance $\ddagger$ : for them it must of necessity lead to the same

* I fear that I bave on previous occasions orally assigned to them a wider importance. It is only the fate of the constituent wave-trains that are near the free period that is undetermined.

+ 'The paper, as its title indicates, is concerned mainly with a brilliant application of groups of undulations to the instantaneous explanation of Fox Talbot's interference bands. In connexion with $\S 6$ it may be remarked that the dynamical relations require that a limited disturbance, travelling in a transparent medium, must consist of compensating positive and negative parts.

$\mp$ Formation of the differential equation for the forms of disturbances that are propagated without change of type shows that, when simple wave-trains possess this property, there are in general no other solutions ; the existence of a wave-group in fact implies the existence of the wavetrain through which it travels.

Plit. Mag S. 6. Vol. 10. No. 59. Nov. 1905. 2 R 
law of prismatic dispersive power as holds by the same argument for a disturbance consisting of uniform trains of simple waves, if the average wave-length of the latter corresponds to this group-velocity.

As these considerations relating to the mode of propagation of pulses apply to both Prof. Schuster's and Prof. Ames's arguments, it will not be superfluous to fortify them by the following quotation from Lord Kelvin*, written in connexion with the features exbibited near the beginning and end of a regular gravitational train of surface-waves travelling on deep water :- "Our present solution shows how rapidly the initial sinusoidality of the head and front of a one-sided infinite procession, travelling right-wards, is disturbed in virtue of the hydrokinetic circumstances of a procession invading still water. Our solution, and the item towards it represented in figs. 6 and 7 , and in fig. 2 of $\S 6$ above, show how rapidly fresh crests are formed. The whole investigation shows how very far from finding any definite 'group-velocity' we are, in any initially given group of two, three, four, or any number, however great, of waves. I hope ... to return to this subject in connexion with the energy principle set forth by Osborne Reynolds, and the interferential theory of Stokes and Rayleigh giving an absolutely definite group-velocity in their case of an infinite number of mutually supporting groups."

It would appear then that there is no certain ground, on the basis of the ideas pertaining to group-velocity, for concluding that a prism is competent to disperse any isolated xthereal pulse, or any series of pulses with absolutely irregular statistics, into a series of simple wave-trains, in a regular manner $t$, as an ideal grating could do, the number of undulations in each train being in that case the number of rulings in the grating or a sub-multiple thereof.

But Lord Rayleigh in his recent paper has thrown fresh light on the subject of the general action of dispersive media, by examining the disturbance that follows an impressed travelling aperiodic pulse, maintained at constant intensity, and showing that such a pulse imitates in some respects closely the behaviour of a wave-train $\ddagger$. He in fact points out the analogy with the surface-waves produced by a boat travelling with uniform velocity on a lake, which, as everybody has

* Kelvin, "On the Front and Rear of a Free Procession of Wares in Deep Water." Phil. Mag. vol. viii. 1904, p. 468.

$\uparrow$ What applies to a prism would probably also apply to colourperception by the eye.

$\ddagger$ A more direct investigation than that quoted from Lord Kelvin's note of 1877 is given in Prof. Lamb's 'Hydrodynamics,' 1895, $\$ 227$. 
observed, are dispersed into simple wave-trains each travelling in its own appropriate direction. It would seem indeed that this illustration bears more closely on the action of a travelling source impressed on the medium than on the fate of an unsupported pulse travelling across it spontaneously. In default of a constant supply of power to the boat, to be spent in making new waves, it would soon lose its velocity unless it had a store of kinetic energy great for its size. Thus this close analogy with ordinary dispersion, which is afforded by the dispersed wave-trains excited by a pulse, impressed and maintained from ontside, appears to leave where it was the question of the fate of an isolated unsupported pulse, propagated into the dispersive medium and then left to itself. The steadiness which in the ordinary dispersion-theory arises from the succession of fresh waves of the train, is obtained in the illustration above by the maintenance of the energy of the pressural source, with results in close analogy in the two cases.

It thus still seems difficult to evade the force of the argument of Sir George Stokes * :- "When you let a ray of light fall on a refracting medium such as glass, motions begin to take place in the molecules forming the medium. The motion is at first more or less irregular; but the vibrations ultimately settle down into a system of such a kind that the regular joint vibrations of the molecules and of the ether are such as correspond to a definite periodic time, namely that of the light before incidence on the medium. 'That particular kind of vibration among the molecules is kept up, while the others die away, so that after a prolonged time-the time occupied by, we will say, ten thousand vibrations, which is only about the forty thousand millionth part of a second-the motion of the molecules of the glass has gradually got up until you have the molecules of the glass and the ether vibrating harmoniously together. But in the case of the Röntgen rays, if the nature of them be what I have explained, you have a constant succession of pulses independent of one another. Consequently there is no chance to get up hasmony between the vibrations of the ether and the vibrations of the body."

The distinction may perhaps be put more definitely. White light from an incandescent solid is made up of a vast number of pulses arising from the molecular shocks incessantly occurring in the hampered spaces to which the molecules are confined. On the other hand, the Röntgen rays are made up

* Wilde Lecture " On the Nature of the Röntgen Rays," 1897. Math. and Phys. Papers, v. p. 271. 
of the independent sporadic shocks transmitted through the æether from the impacts of the separate and independent cathode particles. Both kinds of disturbance are resolvable by Fourier's principle into trains of simple waves. But if we consider the constituent train having wave-length variable between $\lambda$ and $\lambda+\delta \lambda, i$. $e$. varying irregularly from part to part of the train within these limits, a difference exists between the two cases. In the case of the white light the vibrationcurve of this approximately simple train is in appearance steady : it is a curve of practically constant amplitude, but of wave-length slightly erratic within the limits $\delta \lambda$ and therefore of phase at each point entirely erratic. In the Fourier analysis of the Röntgen radiation the amplitude is not regular, but on the contrary may be as erratic as the phase. The origin of this difference is that the body radiating the white light is presumably so far in a steady state that each element of it has a definite temperature at each instant, which implies a statistical uniformity in the vibratory disturbance that is emitted. Or, approaching the subject from the side of the thermodynamics of radiation, each elementary constituent wave-train, say that corresponding to the interval $\delta \lambda$ above, has its own temperature which it carries permanently along with it, the same as the temperature of its source supposed a perfect radiator. This temperature is at each point of it a function of the energy-density, and therefore of the amplitude of the radiation. If the amplitude were different along two reaches of the train, the reach of higher amplitude could be in equilibrium of spontaneous exchanges of energy with a perfect radiator of higher temperature than its own source, and ideal automatic arrangements involving intensification of the energy would be possible, in opposition to Lord Kelvin's fundamental principle of degradation. In the internal equilibrium to which a material system nearly instantaneously settles down, in acquiring a definite temperature for each element of its mass, such differences of amplitude must thus have disappeared : the state of nniform amplitude is, in fact, the most probable one *.

In the distinction which is here suggested, the average degree of suddenness of the Röntgen pulses is not involved. That wonld still be capable of estimation by experiments on diffraction of rays travelling in free ether, in the manner of Haga and Wind. But no physical rationale of prismatic dispersion $\dagger$ except the influence of the vibrations excited in the material system seems now to be entertained; and this

* It is hopéd to pursue this idea in another connexion.

+ Propagation in limited systems such as bars is not to the point. 
appears to impose a limit to the types of disturbances that are subject to regular dispersion.

Even in the case of wave-trains excited on the surface of water by a travelling source, where, as Lord Rayleigh remarks, there is no structural periodicity ${ }^{*}$, the presence of the wave-trains travelling in any direction does, at any rate, depend on the persistence of free periodic trains of waves. If we imagined the water replaced by a medium in which no free wave-trains could travel with velocities within certain limits, then we would expect a gap in the wave pattern formed by the travelling source, corresponding to those limits. The passing remark of Sir George Stokes, which likens the synchronous optical vibration of a transparent solid body to the sonorous vibration of the sounding-board of a pianoforte, thus assigning it to regions of the material medium in bulk rather than to its individual molecules $\dagger$, would bring the optical effect somewhat nearer to the water-wave phenomenon.

But the feature in the case of the water-waves to which Lord Rayleigh doubtless intends to draw special attention, is the absence of gradual initiation and delay of effects ; as soon as the source begins to move with uniform velocity, the wavepattern begins to travel out from it, and as soon as motion of the source stops, so does the formation of the wave-pattern. By a legitimate application of the principle of group-velocity, the number of undulations formed is shown to be connected with the distance over which the source has moved; just as the number of waves formed from a single freely travelling pulse by a grating is determined by the number of its lines over which the pulse has travelled.

At first sight, as above stated, it is difficult to detect sufficient similarity to the optical case. But if we consider (with Lord Rayleigh, p. 404) a thin plane pulse incident from free æether obliquely on an infinitely extended plane face of a refracting medium, the intersection of the pulse with the face will be just such a maintained disturbance, travelling

* Prof. Schuster (Phil. Mag. Jan. 1904) puts the point as follows:"As we may imagine continuous media of such elastic properties as to give dispersion, the true explanation must be independent of the sympathetic vibrations," on which I had relied in 'Ather and Matter,' p. 248. The force of this is obvious: yet, when friction is ruled out, what can there be, as a matter of fact, except conspiring periodicities in time (free periods) or space, to modify simple elastic waves, which travel without change, into the dispersive type?

$\dagger$ In the case of a vapour, the molecules, being isolated, must however operate independently. 
along the face, as we require. If the medium has the dispersive quality fully developed, for all disturbances however sharp, $i . e$. if the differential equation determining dispersive vibration has no limits to its full application to such disturbances, the resolution into trains of waves must be granted as a necessary consequence of the analysis for a steady travelling source.

This rationale of the dispersive refraction, at a plane surface, of an obliquely incident thin plane pulse, is one of those obvious things that when once grasped form a permanent addition to our stock of physical imagery*. One can picture its appication to water-waves. A tract of water may be jmagined, of small uniform depth $h$, in which therefore all waves travel with the same velocity $\sqrt{g h}$, separated from a region of deep water, in which the velocity of a train depends on its wave-length, by a straight boundary. A disturbance consisting of a thin plane ridge can advance obliquely towards the deep water without change of form ; the successive parts of the ridge reach the boundary in the manner of a maintained local disturbance running along the boundary with uniform speed, of which a definite fraction is transmitted across into the deep water, the rest being reflected back. The mode of this transmission is, by symmetry, the same as if that part of the disturbance were doubled and the deep water were unlimited on hoth sides: regular wav-trains are shed off dispersively in the different directions, as in the case of the boat described above, with wave-lengths such that their velocity can just. keep up with the travelling source, while the distribution of intensities between the various directions depends on the character of the moving source, $i$. e. of the incident travelling ridge of water. The waves must, in fact, form a steady pattern travelling with the source : thus the velocity of free propagation of the component train travelling in any direction must be the component in that direction of the velocity of the source. But as the travelling source has finite size, this component train, though nearly homogeneous in wave-length, is not quite so; being nearly homogeneous, the dispersive quality of the medium will make its waves travel in groups, which progress in the known manner with only half the velocity of their component waves. 'Thus after the train is well formed, the groups of disturbance will recede from the

- Lord Rayleigh considers this explanation of the refraction of a pulse into a dispersive medium to be less simple than his first case of the propagation of a plane pulse in such a medium: but owing to the difficulty described above, regarding the maintenance of such a pulse, I have failed to appreciate the argument in that case. 
source with a velocity equal to the difference of these two velocities, and Lord Rayleigh's determination of the length of disturbance emitted in a given time ensues,--subject, however, to the reservation quoted above from Lord Kelvin as regards the extreme head of the train. The account of the process, which is indicated by Lord Rayleigh, seems in its essentials to be fully verified.

Yet the quotation above made from Sir George Stokes, in which the imagery is optical instead of hydrodynamical, appears to show a different aspect of the picture which we are bound to follow out; though Lord Rayleigh has guarded himself against it by his reservation, "so long at least as we are content to take for granted the character of the dispersive medium-the relation of velocity to wave-length-without inquiring further as to its constitution." The postulate thus indicated is that the partial differential equation of propagation is to hold true without limitation. 'This implies that the dispersive medium must be homogeneous in space; if it had minute alternating structure, then this differential equation could not of course be applied without modification to waves of length comparable with the dimensions of that structure, - i circumstance on which Cauchy reared his original attempt at an explanation of optical dispersion. But it requires also that the medium should, so to speak, be homogeneous in time. An optical dispersive medium is made up of elements which have periods of free vibrations of their own, that are more or less durable ; the differential equation will not hold for disturbances whose seale of duration is so small as to be of the same order as the time of the natural subsidence of free disturbance among the elements of the medium. In connexion (originally) with the dynamical theory of viscosity in gases, Maxwell introduced the term time of relaxation to express the time, roughly assignable, that it would take for a local derangement of the molecules of the medium to smooth itself out. In optics it is the time needed for the iree irregular vibrations of an element of the medium, produced by a local shock or other disturbance, to die out by dissipation into surrounding elements. The theory of regular dispersion of a disturbence into wave-trains caused by refraction, re-stated above for the hydrodynamic case of waves on water, cannot be applied in the optical case unless the scale of duration of the disturbance is long compared with the time of optical relaxation of the dispersive medium. In Sir George Stokes's illustration, taken at a venture, the time of relaxation would be ten thousand times the period of a light-wave ; if so, regular refraction and dispersion would 
hardly be established for sequences of less than ten thousand similar waves. Perhaps the only means of even roughly guessing at the time of optical relaxation is by the time-lag in such phenomena as fluorescence, which are connected in part with free internal vibrations excited in the elements 0 . the medium. Stated in the present form, the criterion that a Röntgen æther-pulse should be regularly refracted and dispersed into wave-trains, according to a process of which Lord Rayleigh's rationale has been paraphrased above, is that its duration should be long compared with the time of optical relaxation of the dispersing medium. In the hydrodynamic illustration the restriction does not arise, for the time of molecnlar relaxation is far beneath the period of any observable surface-waves.

To sum up, it now seems clear that Lord Rayleigh's application of the phenomena of a maintained moving source gives an adequate picture of the modus operandi of the dispersion of an incident aperiodic disturbance into regular wave-trains by refraction, for all types of disturbance that are slow compared with the period of natural molecular relaxation of the refracting medium,--provided, however, anomalous dispersion, which cannot be included unless a quasi-frictional term is assumed in the analysis, plays a part which is unimportant. But it is still held to be unilkely that rethereal pulses of the type of the Röntgen rays come as a rule within this limit. If this be so, white light, such as can be regularly dispersed by a prism, cannot consist of wholly irregular æetherenl disturbance; each Fourier component, comprised within say the infinitesimal range of wave-length between $\lambda$ and $\lambda+\delta \lambda$, must have sequences of regularity in its amplitude, of duration comparable with the time of optical relaxation of the dispersing medium.

Cambridge, October 10, 1905 .

LXIV. On the Emission of Negative Corpuscles by the Alkali Metals. By J. J. Thomson, M.A., F.R.S.*

T $\mathrm{T}$ is well known that the alkali metals when exposed to 1 light give out negative corpuscles, even when the light is of very feeble intensity. Thus Elster and Geitel found that the light emitted by a piece of glass rod heated to a dull red heat was sufficient to make rubidium emit corpuscles. It has not, however, as far as I am aware, been noticed that

* Communicated by the Author. 